\title{
Bericht über mein Schulpraktikum in Alice Springs
}

\section{Lisa Wendlandt}

Ausgangspunkt meines Praktikums war die Idee, im Northern Territory, Australiens Wüstenstaat, verschiedene Schulen und Bildungseinrichtungen zu besuchen, um die unterschiedlichen Bildungsprogramme für Aborigines (Kinder und Jugendliche) in der Praxis kennen zu lernen. Motiviert war ich durch mein Interesse an den Sprachen der Aborigines und insbesondere dem Aboriginal Englisch als Beispiel einer KontaktSprache. Vordergründig schien sich mir im Aboriginal Englisch ein so enormer, durch die Kolonisation entstandener Fremdeinfluss zu manifestieren, daß sich der Gedanke an den gleichzeitigen Verlust der kulturellen Identität der Aborigines aufdrängte. Ich hatte aber Untersuchungen zum Aboriginal Englisch gelesen, die erstaunliche Anhaltspunkte für die Annahme einer starken semantischen Kontinuität zwischen Aboriginal Sprachen und dem Aboriginal Englisch lieferten. Dies legte den Gedanken nah, daß es sich weniger um eine Anpassung der Aborigines an die Englische Sprache, als vielmehr der Englischen Sprache an die Kultur und Diskursformen der Aborigines handelt. Dem wollte ich gerne in der Praxis nachgehen und plante zu diesem Zweck, an den verschiedenen Schulen eine Reihe von Tonaufnahmen des Aboriginal Englisch zu sammeln.

Darüber hinaus waren für mich auch die historischen Entstehungsbedingungen des Aboriginal Englisch während der Kolonialzeit von großem Interesse. Im Sommersemester 2001 hatte eine Exkursion zu den Archiven der Herrnhuter Brüderunität in Sachsen mein Interesse für die Australienmissionen des 19. und 20. Jahrhunderts geweckt. Die Herrnhuter Archive erlaubten mir erste interessante Einblicke in die Art der Begegnung und des Sprachkontaktes zwischen den Herrnhuter Missionaren und den Aborigines. Nun hoffte ich, während meines Praktikums in Australien einige der ehemaligen Missionsstationen besuchen zu können und in Archiven die Nachforschungen zur Geschichte des Sprachkontakts während der Missionierung fortsetzen zu können.

\section{Erste Eindrücke vor Ort}

Als der Zug nach einer langen Nachtfahrt unter sternenklarem Wüstenhimmel in Alice Springs einrollte, überkam mich zum ersten Mal das Gefühl der Beklommenheit. Barfuss standen sie in der Landschaft, in der Ebene vor den Hügeln der Stadt, neben den Schienen des Zuges und in den Vorgärten ihrer kleinen Häuser. Hier, aus dem Fenster des fahrenden Abteils blickend, sah ich zum ersten Mal die Aborigines in ihren Randsiedlungen vor Alice Springs. Ich fühlte mich fehl am Platz wie ein Eindringling, unerwünscht und ungebeten. Auch in der Innenstadt legte sich dies Gefühl kaum. Der Weg vom Bahnhof zur Jugendherberge führte über das ausgetrocknete Flussbett des Todd River, in dessen weißem Flussbett außer den Aborigines niemand sonst im Schatten der Eukalyptusbäume saß. Mein erster Erkundungsgang in die Fußgängerzone im Zentrum - zurück über die Brücke des Todd River - führte mich mitten in eine Welt, deren Regeln ich noch nicht verstand: Eine getrennte Welt, in der hier die Touristen und "Weißen ${ }^{3}$ in den Cafés speisten oder Einkäufe machten, und dort Gruppen von Aborigines auf den Bänken und im Gras saßen, viele betrunken, manche grölend und schimpfend, wenige ihre Kunst feilbietend. Das Aufeinandertreffen der Kulturen und seine Konsequenzen bestimmte die Atmosphäre der ganzen Stadt und hatte bei allem eine unwirkliche Alltäglichkeit angenommen. Der Konflikt einer ungelösten Geschichte 
schien offen und zugleich wortlos zutage zu liegen. Ich war überrascht, verunsichert und voller Fragen.

\section{Ein Klassenzimmer in der Stadt}

Meine Zeit in Alice Springs begann mit dem Besuch der Braitling Primary School, einer städtischen Grundschule, in der 25\% der SchülerInnen Aborigines sind. Durch ein besonderes Betreuungsprogramm gibt es täglich Einzel- und Kleingruppenunterricht nur für die Aboriginal Kinder, die aus dem grossen Klassenverband herausgelöst und von speziellen Lehrkräften unterrichtet werden. Die Unterrichtssprache ist ausschließlich Englisch. Bei meiner Ankunft waren gerade zwei sehr kleine Aboriginal Mädchen in die Vorschulklasse gekommen, die sich miteinander in Arrernte unterhielten, einer in Alice Springs weit verbreiteten Aboriginal Sprache. Ihre Englischkenntnisse waren minimal, und auch in den höheren Klassen begegneten mir viele, deren Englisch weit unter dem Niveau der gleichaltrigen europäisch-australischen Kinder lag. Natürlich darf man sich die Aboriginal SchülerInnnen nicht als eine homogene Gruppe vorstellen, sondern als Kinder ganz unterschiedlicher Herkunft, Hautfarbe und Sprache. Sie kamen aus der Stadt oder den Towncamps (provisorischen Randsiedlungen), aus ein- oder mehrsprachigen Haushalten, in dene Englisch, Warlpiri, Arrernte oder andere Aboriginal Sprachen gesprochen wurden. Manche hatten wohlsituierte, berufstätige Eltern, andere kamen aus zerrütteten, von Drogenproblemen geprägten Familienverhältnissen.

Generell beobachtete ich eine recht angespannte Stimmung unter den Aboriginal SchülerInnen, auch und gerade in den speziell betreuten Einzelsitzungen, spürte das Bemühen und den Druck der Lehrerinnen, ihre Erwartungshaltung und auch ihre Hilflosigkeit. Der Leistungsdruck, der ja strukturell mit der Institution Schule verbunden ist und den potentiell alle Kinder einmal erfahren, besteht nochmal in ganz anderer Qualität für die Aboriginal Kinder. Es wäre $\mathrm{zu}$ fragen, ob und inwieweit die hohe Abwesenheitsrate der Aboriginal SchülerInnen mit dem Gefühl der Überforderung und des Leistungsdrucks in Verbindung steht, oder ob sie eher auf außerschulische Faktoren wie familiäre Umstände u. ä. zurückzuführen ist. In Gesprächen mit der Direktorin und verschiedenen LehrerInnen der Schule wurde mir schnell klar: Die mangelnde, bzw. unregelmäßige Anwesenheit wird als das Hauptproblem in der Ausbildung und Integrierung der Aboriginal SchülerInnen angesehen. Mühsam wir da einem Jungen das Alphabet beigebracht, der kurz darauf für sechs Wochen nicht mehr erscheint, weil er seinen Großvater nach Adelaide begleiten muss, eine neue Autobatterie für den Toyota besorgen, und wenn er dann wiederkommt, hat er das ganze Alphabet vergessen. Welche Frustration das für die LehrerInnen bedeutet! Ganz von vorne anfangen, und immer freundlich... Und welche Frustration für den Jungen, der nicht weiß, warum er in so enttäuschte Gesichter gucken muss, und welche Bedeutung das überhaupt für ihn haben soll, das A,B,C.... !

Der entscheidende Punkt ist, dass die Schule im wesentlichen auf die Kooperation der Eltern angewiesen ist - die Eltern aber, nicht selten Analphabeten, sehen oft keinen Sinn darin, ihre Kinder zur Schule zu schicken. Interessant ist in diesem Zusammenhang das "Großmütter-Phänomen³: Zum Teil sind es die Großmütter in den Aboriginal Familien, die die Verantwortung für die schulische Ausbildung ihrer Enkelkinder übernehmen, sie zur Schule bringen, die Hausaufgaben mit ihnen machen, den Kontakt zur Schule pflegen, etc. Sie gehören zu einer Generation, die oft noch unter dem Einfluss der Missionsstationen eine Schulbildung von relativ hohem Niveau durchlaufen hat. Dagegen 
können viele ihrer eigenen Kinder (die Eltern der heutigen GrundschülerInnen) weder Lesen noch Schreiben. Diese "Bildungslücke ${ }^{3}$ halte ich für ein interessantes historisches Phänomen, das sicherlich im Zusammenhang mit der Self-determination-Ära der 70er Jahre gesehen werden muss. Für manche der heutigen GrundschülerInnen stellen die alphabetisierten Großeltern tatsächlich die einzige Brücke zur westlichen Schulbildung dar, weshalb die Generation dieser "Ältesten ${ }^{3}$ in ihrer verbindenden Funktion nicht unterschätzt werden sollte.

\section{Ein Klassenzimmer in der Luft}

Das Großmütter-Phänomen war auch von einiger Bedeutung in der Alice Springs School of the Air, die ich als nächstes besuchte. In dieser "Luftschule 3 werden jene Kinder via Funkübertragung unterrichtet, die weit übers Land verstreut auf abgeschiedenen Farmen oder in winzigen Kommunen wohnen, hunderte und sogar tausende Kilometer von Alice Springs oder der nächsten Schule entfernt. Auch in dieser Luftschule gibt es Klassenverbände, und die Aboriginal SchülernInnen werden als gesonderte Gruppen unterrichtet. $\mathrm{Zu}$ festgelegter Uhrzeit hofft die Lehrerin in der Funkzentrale von Alice Springs, alle SchülerInnen an Ihren hauseigenen Funkgeräten zu erreichen.

"Shane, are you there?" Stille. "Hallo? Can you hear me?" Stille. Warten. Nur ein Knistern im Radio. Nein, Shane ist heute nicht da. Die anderen Kinder mischen sich ein, ihre kleinen Stimmen dringen aus dem Äther, oft verzögert, oft unverständlich. Shane sei bei seiner Tante, glauben sie zu wissen, sie, die sie hunderte von Kilometern von Shane entfernt leben. Shane jedenfalls ist nicht da, und das ist nicht allzu ungewöhnlich. Auch die School of the Air ist fundamental auf die Kooperation und die Unterstützung von Seiten der Eltern angewiesen. Wie aber sollen Eltern motiviert werden, deren Leben oft so völlig anders ausgerichtet ist, die im Familienzusammenhang gut ohne Englisch auskommen und gelernt haben, einem Bildungssystem zu mistrauen, dass für sie nicht zur erhofften sozialen und ökonomischen Integration geführt hat? Dennoch bleibt die westliche Schulbildung scheinbar reizvoll genug, damit ein paar Großeltern, Eltern und ältere Geschwister die kleineren Kinder bei ihren täglichen Funkstunden und Schularbeiten betreuen. Die vollgeschriebenen Hefte mit den Geschichten, Übungen und Hausaufgaben treten dann den langen Postweg nach Alice Springs an, um dort von der Lehrerin korrigiert zu werden. Manchmal holt die Lehrerin sie auch selbst ab, denn alle paar Wochen fährt sie jedes einzelne Kind bei sich zu Hause besuchen und verbringt ein bis zwei Tage mit der Familie.

Absurder Weise hat diese Form des Unterrichts, wie er durch die School of the Air geleistet wird, trotz der enormen geographischen Entfernungen einige Vorteile gegenüber der Lernsituation in einer städtischen Grundschule wie der Braitling Primary School: Vielleicht, weil die Kinder von zu Hause aus lernen können und direkt von einem Familienmitglied begleitet werden. Die betreuenden Familienmitglieder (die sogenannten "TutorInnen") sitzen während des Funkunterrichts neben den Schülerinnen. Während die Lehrerin per Funk mit den Kindern spricht, gibt sie hin und wieder ganz direkte Anweisungen und Vorschläge an die TutorInnen, die auf diese Weise eng in den Lernprozess einbezogen werden. Es entsteht somit nicht nur zu den SchülerInnen, sondern auch zu ihren Eltern oder Familienangehörigen ein enges Vertrauensverhältnis, das durch den regelmäßigen Besuch in den Kommunen noch verstärkt wird. Diese Ausbildungsform entspricht in vieler Hinsicht eher den traditionellen Lehr- und Lernstrukturen der Aborigines, für die die Familie eine extrem hohe Rolle spielt und 
Wissen stets innerhalb der Familie weitergegeben und autorisiert wurde. Man darf jedoch nicht vergessen, dass diese Form der familienintegrierten und sehr persönlichen Schulbildung im australischen Gesamtkontext eine Ausnahmesituation darstellt und von den meisten innerstädtischen Schulen aus strukturellen Gründen nicht zu leisten ist. Während die Braitling Primary School mit einem Klassenumfang von 15-25 SchülerInnen arbeitet, besteht die Aboriginal Klasse der School of The Air durchschnittlich aus 6-8 SchülerInnen. Auch ist es sicherlich von Bedeutung, daß sich in dieser Klasse ausschließlich Aboriginal Kinder aus abgelegenen Kommunen befinden, die aus relativ ähnlichen Lebensverhältnissen und Sprachzusammenhängen kommen. Mit anderen Worten, die Kinder teilen viele ihrer eigenen kulturellen Erfahrungen miteinander, ohne ständig unter dem Einfluss der "whitefella kids" (der weißen Kinder) mit dem Gefühl des Anders-Seins und der Benachteiligung konfrontiert zu werden. Erfahrungen wie diese stellen die in den letzten Jahrezehnten enthusiastisch hervorgehobenen Vorteile der Koedukation von aboriginal und europäisch-australischen Kindern zumindest in Frage. Trotz der politischen Ideologisierung, die die Koedukationsdebatte erfahren hat, halte ich es für unumgänglich, die durchaus kontraproduktiven Auswirkungen so manchen Koedukationsprogramms ernsthafter zu bedenken, wie dies in einigen jüngeren Theorien bereits getan wird. Kinder, die nicht zur Schule kommen, kann man nicht aufnehmen.

An einem windstillen Tag mit $40^{\circ}$ Celsius im Schatten besuchte ich zu guter Letzt die Grundschule in Amoonguna, einer Aboriginal Siedlung ca. 70 Kilometer von Alice Springs entfernt. Die Zustände hier übertrafen alles, was ich zuvor erlebt hatte. Noel, ein alter Pionier im Bereich Aboriginal Education und jahrzehntelanger Schullehrer in diversen Kommunen des Northern Territory, war an diesem Tag als Aushilfslehrer der Schule im Dienst und hatte mich in Alice Springs mit dem Auto abgeholt.

Als wir um halb neun die Stufen des auf Stelzen gebauten Schulhauses emporstiegen, war weit und breit noch niemand zu sehen. Noel schlenderte in die Küche um zu sehen, ob das Frühstück für die Kinder geliefert worden war. Als Teil des Regierungsprogramms für die Amoonguna School wird täglich ein kostenfreies Frühstück an die SchülerInnen verteilt mit dem Ziel, das Bewusstsein für gesunde Ernährung in der Kommune zu erhöhen. Meiner Ansicht nach handelt es sich bei diesem Frühstück um ein relativ kritisches Unterfangen, entlässt es doch die Eltern und Familien auf gewisse Weise aus einer der wenigen, noch in ihrem Verantwortungsbereich liegenden sinnerfüllten Aufgaben, nämlich der Sorge um die eigenen Kinder. Darüber hinaus impliziert es ein Mistrauen in ihre Fähigkeiten als Eltern und kann leicht als Bevormundung und Konkurrenz verstanden werden. Über das schlichte Verteilen der Butterstullen und Äpfel hinaus wird in Amoonguna keine konstruktiv-aufklärerische Arbeit geleistet, bei der die Verantwortung bei den Eltern verbleiben müsste und die angestrebte Veränderung (in Richtung einer gesünderen Ernährung) von den Eltern selbst als sinnvoll erkannt und initiiert würde. Das Frühstück aber war geliefert worden, und nun war es an der Zeit, die Schulglocke zu läuten. Ihr Klang schallte über die ganze Siedlung, und Noel und ich hockten uns in das Klassenzimmer und warteten. Nichts geschah. Vielleicht kommt ja heute keiner, sagte Noel lachend. Das wäre nicht ungewöhnlich. Viertel nach Neun ein neuer Versuch: Wieder hallte der Klang der Glocke über die Siedlung. Um halb zehn trudelten ein paar Kinder ein, barfuss lärmten sie mit einem kleinen Hund in die Klasse. Viele waren es nicht, vielleicht fünf oder sechs 
Mädchen und Jungen im Alter von sechs bis neun. Der Hund musste draußen bleiben und Noel begann mit dem Unterricht. Um 10 Uhr ging die Tür auf, ein paar Jungen stießen dazu, wollten auch noch mitmachen. Von einigen Kindern kannte Noel nicht einmal die Namen, und andere hatte er schon wochenlang nicht mehr gesehen. Ihr Erscheinen ist mehr oder weniger unregelmäßig, und demnach klafft der Wissenstand der einzelnen SchülerInnen zum Teil auch ganz beträchtlich auseinander. Dass aber an diesem Tag überhaupt einige von ihnen zum Unterricht erschienen, versetzte Noel beinahe in Erstaunen: Am Tag zuvor war nämlich mal wieder der monatliche Zahltag der "Welfare Pensions ${ }^{3}$ gewesen. Der Hausmeister der Schule erzählte uns, wie er das gesamte Dorf in sechs proppenvollen Ladungen mit seinem Kleinbus nach Alice Springs fahren musste, wo alle ihr Geld abholten und sich den Abend mit Alkohol und Pokerspielen zu versüßen gedachten. Viele kamen gar nicht zurück an diesem Abend, sondern übernachteten in der Stadt. Andere kamen sehr spät und sehr betrunken nach Amoonguna zurück, im Bus des sehr treuen Hausmeisters, der einer der wenigen mit Führerschein im Dorf ist. Die wenigen Kinder, die zur Schule kamen, hatten tatsächlich noch nicht gefrühstückt. Aber ob das wirklich an einem Mangel an Fürsorge der durchnächtigten Eltern lag oder vielmehr an der intelligenten Gewissheit, ihre Kinder in der Schule ja gut versorgt zu wissen, das muss wohl unbeantwortet bleiben. Die Amoonguna Primary School, die 1955 als Teil des Assimilationsplans der Regierung gebaut wurde, umfasste in den 60ern noch 120-130 Schülerinnen und Schüler. Heutzutage erscheinen täglich, wenn überhaupt, nur noch 10 bis 20 Kinder zum Unterricht. Auch das kostenlose Frühstück vermag daran nichts zu ändern. Wegen Ineffizienz wird derzeit überlegt, die Amoonguna School zu schließen und die wenigen Kinder lieber mit dem Bus in die städtischen Schulen zu fahren. Für mich spitzte sich hier in Amoonguna die Frage zu, die sich mir auch schon an den anderen Schulen gestellt hatte: Wie sollte ich Kinder aufnehmen, die gar nicht oder nicht regelmäßig zur Schule kamen? Die Ausmaße jener fundamentalen Krise, in der sich die australischen Aborigines noch immer und vielleicht mehr denn je befinden, überlagerten meine linguistischen Fragen. Dennoch habe ich insgesamt über 300 Minuten digitales Tonmaterial der Schülerinnen und Schüler sammeln können, darunter einige prägnante Beispiele des Aboriginal Englisch mit seinen typischen, ihm zugeschrieben Merkmalen, sowie Aufnahmen von Freizeit-Situationen auf dem Pausenhof in Amoonguna, in denen sowohl Englisch als auch eine oder mehrere Aboriginal Sprachen (die ich noch nicht habe identifizieren lassen) parallel gesprochen werden.

\section{Archivarbeit und neue Projekte}

Unkomplizierter gestaltete sich mein Vorhaben der Archivarbeit zur Geschichte des Kontakts zwischen Missionaren und australischen Aborigines. Statt weitere Schulen zu besuchen, knüpfte ich Kontakt zum Strehlow Research Centre, wo ein Projekt für mich geradezu vom Himmel fiel - so kam mir das jedenfalls vor, denn ich war ausgehungert nach einer produktiveren Erfahrung. In dem Projekt geht es um die Übersetzung des einzig erhaltenen Kindertagebuchs von Theodor Strehlow, dem Sohn eines deutschen lutheranischen Missionars, der hier in Herrmansburg (ein paar hundert Kilometer südöstlich von Alice Springs) inmitten der Arrernte Bevölkerung aufgewachsen ist, fließend Deutsch und Arrernte zu sprechen lernte und später das Erbe seines Vaters übernahm und transformierte, nämlich die Aufgabe, Sprache und Kultur der Arrernte aufzuzeichen und somit zu "erhalten". Theodor wurde später von den Aborigines auserwählt, als "einer von ihnen" ihre rituellen Gegenstände aufzubewahren, da sie den Untergang ihrer Kultur 
fürchteten. Strehlow war in dieser Funktion einzigartig, und seine Geschichte ist hochinteressant. Natürlich wurde er auch viel kritisiert und seine Mittlerfunktion hat ihn oft in heikle Situationen und Loyalitätskonflikte gebracht. Er hat enorm viele eigene Schriften sowie kulturelle Gegenstände der Arrernte hinterlassen, die heute im Strehlow Research Centre in Alice Springs aufbewahrt wird. Dort bin ich, wie gesagt, auf das in Deutsch verfasste Kindertagebuch aufmerksam gemacht worden, das er 1922 (im Alter von 14 Jahren) geschrieben hat. Es gibt noch keine Übersetzung ins Englische. Viel später, 1969, hat Strehlow dann seinen einzigen Roman veröffentlicht, "The Journey to Horseshoe Bend", in dem er sich ausgerechnet auf das Jahr 1922 bezieht (von dem ja das Tagebuch noch existiert)! Es ist nämlich das Jahr, in dem sein Vater gestorben ist. Ich werde nun das Tagebuch übersetzen, um dann einen literarischen Vergleich zwischen dem Tagebuch und dem Roman zu versuchen. Gibt es ein Zusammenspiel zwischen den beiden Perspektiven auf ein und dasselbe Jahr? Gibt es ein Zusammenspiel zwischen der Form des Tagebuchs und der des Romans? Gibt es Hinweise auf die Schlüsselfrage nach der "Erbschaft" des Vaters aus der Perspektive des Kindes der Gegenwart und der des gereiften Mannes, der zurückblickt?

\section{Interview mit meiner neuen Familie}

Während meiner Zeit konnte ich die Arbeit von David und Bess Price, den Jajirdi CrossCultural Consultants, kennenlernen (vgl. das Interview oben). Ihre Consultancy bietet Kurse in interkultureller Kommunikation, Walpiri-Seminare und Übersetzungsarbeiten an. Spezialisiert hat sie sich auf interkulturelles Training in Arbeitsumfeldern wie z.B. den Goldminen der Tanamiwüste, die sie regelmäßig besuchen. Mit David, Bess und der Tochter Jacinta verbindet mich seit meinem Besuch eine tiefe Freundschaft, und sie haben mich auf Warlpiri-Art in ihre Familie aufgenommen. Das Warlpiri-System zur Klassifizierung von Verwandtschaftsverhältnissen ist ganz anders und komplexer als das unsere. In kreisförmiger, nicht linearer Abfolge stehen da die acht "Skin Names" (Verwandtschaftsnamen) miteinander in Beziehung: "Nakamarra" kann da nicht nur die Mutter von "Nun-garrayi" sein, sondern zugleich auch ihre "Großenkelin". Für mich war das alles sehr verwirrend, und es hat eine Weile gedauert, bis ich ungefähr verstand, was es heißt, dass ich jetzt eine "Nampijinpa" bin. Mit den "Skin Names" sind nämlich auch Verhaltensregeln verbunden, die genau festlegen, wie man sich mit den Vertretern anderer Verwandtschaftsgruppen zu benehmen hat. Durch den Nampijinpa-Status bin ich jedenfalls zur "Tochter" von David und Bess geworden und zur "Mutter" etlicher Kinder, unter anderem der zwei reizenden kleinen Söhne meiner "Schwester" Jacinta. 